\title{
Research on Fault Handling of Cold Chain Logistics Equipment Based on Angle Cosine
}

\author{
Xiaomin Guo*, Yamin Zhao ${ }^{1}$, Jianhua Zhang ${ }^{1}$ \\ ${ }^{1}$ School of Management Engineering, Zhengzhou University, Zhengzhou 450001 China
}

Keywords: case similarity, logistics equipment failure, view matching, entropy weight method, angle cosine

\begin{abstract}
The rapid development of cold chain logistics has led to the introduction of more and more advanced cold chain logistics equipment, so the maintenance and processing of advanced logistics equipment failure has been paid more and more attention. Therefore, this paper presents a case similarity matching algorithm for cold chain logistics equipment based on entropy weight method and angle cosine value based on case-based reasoning. Firstly, the failure parameters of the cold chain logistics equipment are collected, and the objective weight value of the attribute parameters of each case is obtained by using the entropy method. Finally, his weight value and the cosine angle are used to calculate the similarity between cases, and find out the case similar to the new case in the case library. The algorithm is based on the data itself and the manual experience in determining the weight value of the case attribute, and avoids the problem of setting the attribute weight by relying on the knowledge of artificial experience.

\section{INTRODUCTION}

People's living standards are increasing, greatly promoted the development of cold chain logistics, incomplete statistics, China's frozen food production to $20 \%$ of the rate of increase in recent years even $35 \%$ of the high speed increase, much higher than the global $9 \%$ Of the average growth rate (Benhong Peng, 2017),

In the field of logistics, there are few cases of casebased reasoning, and the traditional attribute weights are mostly subjective methods determined by experts, and subjective empowerment law will be biased by the limitations of expert subjective view. Therefore, based on the case-based reasoning, this paper uses the objective weight weighting method, that is, the entropy weight method and the angle cosine combination of cold chain logistics equipment fault handling system.
\end{abstract} making the cold chain logistics system is also getting higher and higher intelligence. The advanced cold chain logistics equipment is also emerging, its functional structure is complex, but the advanced cold chain logistics equipment fault diagnosis and processing, mostly rely on manual diagnosis, not only timeconsuming and accuracy is not high, low efficiency, The degree of hinder the introduction of new cold chain logistics equipment.

The core idea based on case-based reasoning is to solve the new problem according to the characteristic attribute of the problem. It has been applied to health care services (Shijiang Li, 2016), decision support (Yingju Zhang, 2016), fault diagnosis (Shengting Yang,2015), industrial (Xianchao Guo,2016); and other issues to solve the problem. Bingyang Luo designed a locomotive fault diagnosis system based on case-based reasoning technology, which simplifies the diagnosis operation and improves the diagnostic efficiency (Bingyang Luo, 2015). Yan Jun will apply the case promotion technology to the automobile fault diagnosis system to realize the intelligent (AHP) to determine the attribute weight, and give the application example (Jun Yan, 2017); Wang Hao applied CBR to the diagnosis of rotating machinery fault system, the use of the Analytic Hierarchy Process (AHP) to determine the weight of the property, and gived the application examples (Hao Wang, 2017);In order to realize the reuse of multi-similarity fault samples, Guangbin Zhang proposes an empirical learning improvement based on historical data(Guangbin Zhang, 2015

\section{RESEARCH ENVIRONMENT}

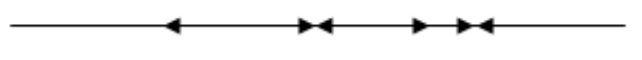

Fig.1 Case reasoning schematic diagram

Based on case-based reasoning, this paper studies the diagnosis and processing of cold chain logistics equipment failure. Case-based reasoning is an important knowledge-based problem solving and learning method in the field of artificial intelligence, with good scalability and portability and self-learning ability. The basic principle of the fault diagnosis system based on casebased reasoning is that the fault events that have been successfully solved are stored in the form of cases. Each case includes condition attributes, decision attributes and solutions. The case form can be described as: Example Case template: $=<$ Attribute list, resolution target, solution, effect evaluation [, sub-case list] [, case example list]>, when a new fault occurs, enter the new 
fault condition value attribute value, and then in the history case library Retrieve the cases that are most similar to the case to be resolved, and adapt to the troubleshooting case based on the solution of the largest similarity case retrieved. The schematic diagram shown in Figure 1.

\section{FAULT DIAGNOSIS SYSTEM FOR COLD CHAIN LOGISTICS EQUIPMENT}

In the fault diagnosis system of the cold chain logistics equipment, the input terminal is the fault attribute value of the cold chain logistics equipment to be solved. The entropy method is used to calculate the condition attribute value and decision attribute value of each fault case in the corresponding historical case library. Attribute weight calculation module and angle cosine value to calculate the historical fault case and the case of the similarity of the case to be solved. The output is the similarity between each historical fault case and the case to be solved.

\subsection{Entropy Weight Method to Determine Attribute Weights}

To calculate the similarity between the historical failure case and the case to be solved, first determine the view of the fault case, that is, the attribute weight vector of the cold chain logistics equipment failure. Entropy method is an objective attribute weight determination method, which avoids the adverse effect of subjective weighting on the result, and can calculate the similarity between the existing case and the target case in the case database more accurately. Therefore, based on the idea of view optimization, the entropy weight method is used to calculate the weight of each case of the fault case when designing the similarity algorithm of case view of cold chain logistics equipment.

Entropy is a measure of the degree of system disorder, the greater the information, the smaller the uncertainty, the smaller the entropy; in turn, the greater the entropy, the greater the uncertainty, the attribute contains the amount of information small. Application entropy can measure the amount of information contained in the data of the cold chain logistics equipment case, and to determine the weight of each aspect. The steps to determine the weight of the entropy method are as follows(Zhanhai Lu, 2012.):

1) Construction of dimensionless cold chain logistics equipment fault value matrix. Assuming that there are ${ }^{m}$ cases to be detected,each case has $n$ attributes that aspect, ${ }^{{ }^{i j}}$ represents the attribute value of the ith case under the jth attribute.Constructs the matrix $V=\left(v_{i j}\right)_{m \times n}$ according to the attribute values,According to the attribute value to build the matrix, because each attribute in the dimension, numerical and other aspects of the differences, so the need for the property value of the dimensionless processing. Get the standardized matrix $R=\left(r_{i j}\right)_{m \times n}$;

2) Calculate the attribute value of the jth attribute of the ith case.which is:

$$
h_{i j}=\frac{r_{i j}}{\sum_{i=1}^{m} r_{i j}}, 0 \leq i \leq m
$$

3) Calculate information entropy and utility values. For the jth aspect, the entropy ${ }^{e_{j}}$ of the aspect is calculated using the aspect values in this aspect, which is, The entropy of the jth is

$$
e_{j}=-k \sum_{i=1}^{m} h_{i j} \ln h_{i j}, j=1,2, \cdots n, k=\frac{1}{\ln n}
$$

4) Calculate the weight of each attribute:

$$
P\left(p_{j}\right)=\frac{1-e_{j}}{m-\sum_{i=1}^{m} e_{j}}
$$

\subsection{Calculate the similarity of the angle cosine}

The cosine of cosine is also called cosine similarity, which is used as a measure of the magnitude of the difference between two individuals in the cosine of the angle between two vectors in the vector space. Each case attribute is considered as a dimension in the multidimensional space when calculating the similarity between the historical failure cases of the cold chain logistics equipment and the case to be solved. For example, the history of the cold chain logistics equipment case set $C=\left\{c_{1}, c_{2}, \cdots, c_{n}\right\}$ has $n$ cases, each fault case $c_{n}=\left(a_{n}^{1}, a_{n}^{2}, \ldots, a_{n}^{m}\right)$ has $m$ attributes, then the case where the dimension is $\mathrm{m}$, the $\mathrm{n}$ fault cases are distributed in the m-dimensional space in different locations From the origin of the vector. The angle cosine is the angle between the fault vector and the historical fault vector in the m-dimensional space, which is the similarity of the two cases. The cosine angle focuses on the difference between the target case and the historical case. Based on the traditional angle cosine algorithm, this paper adds the attribute weights given by entropy weight method to calculate the similarity of fault view of cold chain logistics equipment.

Traditional angle cosine algorithm:

$$
\cos \left(c_{q}, c_{j}\right)=\frac{\vec{c}_{q} \cdot \vec{c}_{j}}{\left\|\vec{c}_{q}\right\| \cdot\left\|\vec{c}_{j}\right\|}=\frac{\sum_{k=1}^{m} v_{q}^{k} v_{j}^{k}}{\sqrt{\sum_{k=1}^{m} v_{q}^{k^{2}}} \cdot \sqrt{\sum_{i=1}^{m} v_{j}^{k^{2}}}}
$$

Weighted cosine similarity algorithm:

$$
\cos \left(c_{q}, c_{j}\right)=\frac{\sum_{k=1}^{m} w_{k}^{2} v_{q}^{k} v_{j}^{k}}{\sqrt{\sum_{k=1}^{m} w_{k}^{2} v_{q}^{k^{2}}} \cdot \sqrt{\sum_{i=1}^{m} w_{k}^{2} v_{j}^{k^{2}}}}
$$




\section{CASE ANALYSIS}

Taking a circuit board fault repair of a cold chain logistics equipment as an example, the fault information includes a set of conditional attribute set and decision attribute set, and selects a set of sub instance cases related to the fault to be solved. As shown in Table 1, The case is:

$$
C_{q}::=\left\{v_{a_{c 1}}: 323 ; v_{a_{c 2}}: 514 ; v_{a_{c 3}}: 472 ; v_{a_{c 4}}: 139 ; v_{a_{d 1}}: \text { null }\right\}
$$

Users by measuring the logistics equipment circuit board at different locations to determine the voltage value of the circuit board type, so as to take appropriate measures.

Table 1 Circuit board failure case aspects of extreme aspects

\begin{tabular}{cclllc}
\hline Fault ID & $\begin{array}{c}\text { The } \\
\text { point } \\
\text { A/mv }\end{array}$ & $\begin{array}{c}\text { The } \\
\text { point } \\
\text { A/mv }\end{array}$ & $\begin{array}{c}\text { The } \\
\text { point } \\
\text { A/mv }\end{array}$ & $\begin{array}{c}\text { The } \\
\text { point } \\
\text { A/mv }\end{array}$ & $\begin{array}{c}\text { The } \\
\text { point } \\
\text { A/mv }\end{array}$ \\
\hline cz025 & 872 & 732 & 910 & 859 & III \\
cz009 & 425 & 273 & 59 & 417 & I \\
cz013 & 247 & 115 & 179 & 653 & III \\
Cz016 & 863 & 233 & 488 & 772 & I \\
cz033 & 226 & 309 & 221 & 126 & II \\
cz028 & 682 & 195 & 566 & 435 & II \\
Cz011 & 531 & 263 & 648 & 540 & I \\
\hline
\end{tabular}

The problem of the fault cases and the target cases shown in Table 1 are discretized and the fault ID is converted into the new value of the domain of the knowledge expression system. The results are shown in Table 2:

Table 2 circuit board fault knowledge expression system

\begin{tabular}{cccccc}
\hline$U$ & $a_{c 1}$ & $a_{c 2}$ & $a_{c 3}$ & $a_{c 4}$ & $a_{d 1}$ \\
\hline$C_{q}$ & 1 & 1 & 2 & 2 & null \\
\hline$C_{1}$ & 3 & 3 & 2 & 3 & I \\
$C_{2}$ & 2 & 1 & 1 & 2 & II \\
$C_{3}$ & 1 & 1 & 1 & 2 & II \\
$C_{4}$ & 3 & 1 & 2 & 3 & I \\
$C_{5}$ & 1 & 1 & 1 & 1 & III \\
$C_{6}$ & 3 & 1 & 2 & 2 & I \\
$C_{7}$ & 2 & 1 & 2 & 1 & III \\
\hline
\end{tabular}

The weights of each attribute are calculated by entropy weight method as follows:

Table 3 Entropy weights calculated by the weight of each attribute

\begin{tabular}{clcc}
\hline P1 & P2 & P3 & P4 \\
\hline 0.2314 & 0.3248 & 0.2325 & 0.2113 \\
\hline
\end{tabular}

The similarity between the historical case and the target case is as follows.

By comparing the similarity between the Euclidean distance and the cosine angle, we get the maximum similarity between the $C_{3}$ in the case database and the case to be solved. At this time, enter the condition part of the problem to be solved. Give its solution value, that is:

$$
C_{q}::=\left\{v_{C q_{-} a_{c 1}}: 323 ; v_{C q_{-} a_{c 2}}: 514 ; v_{C q_{-} a_{c 3}}: 472 ; v_{C q_{-} a_{c 4}}: 139 ; v_{C q_{-} a_{d 1}}: I I\right\} \text {, }
$$

Cold chain logistics equipment maintenance staff can be type II corresponding maintenance method to start the repair process.

Table 4 The similarity between the target case and each historical case

\begin{tabular}{cccc}
\hline$U$ & $\cos \left(c_{q}, c_{j}\right)$ & $\operatorname{sim}\left(c_{q}, c_{j}\right)$ & Similarity sort \\
\hline $\mathrm{C}_{1}$ & 0.8191 & 0.9095 & 7 \\
$\mathrm{C}_{2}$ & 0.9231 & 0.9616 & 4 \\
$\mathrm{C}_{3}$ & 0.9648 & 0.9824 & 1 \\
$\mathrm{C}_{4}$ & 0.9353 & 0.9676 & 3 \\
$\mathrm{C}_{5}$ & 0.9462 & 0.9731 & 2 \\
$\mathrm{C}_{6}$ & 0.9079 & 0.9539 & 5 \\
$\mathrm{C}_{7}$ & 0.8994 & 0.9497 & 6 \\
\hline
\end{tabular}

\section{CONCLUSIONS}

In view of the traditional cold chain logistics equipment failure maintenance is not very good intelligence, only to stay in the manual experience to judge, maintenance personnel professional knowledge requirements, hinder the advanced cold chain logistics equipment, can not effectively meet the current cold chain logistics system Based on the analysis of the related literature, based on the CBR system, this paper establishes the intelligent entropy weight method and the angle-of-cosine value of the cold chain logistics equipment fault case similarity matching algorithm The And the validity of the method is verified by an example. No need to build a detailed model can be fault diagnosis. Its high flexibility and operability can make the cold chain logistics equipment diagnosis system more efficient and accurate, to avoid the inefficient manual experience in the diagnosis process for the cold chain logistics equipment and the development of a good foundation. But for the maximum similarity case still can not meet the maintenance needs of the correction and storage process need further study.

\section{ACKNOWLEDGEMENTS}

This work is supported by innovative Development Fund of Zhengzhou University School of Management Engineering (20170604) and the Excellent Young Teacher Development Fund Project of Zhengzhou University (Foundation No.2015SKYQ15).

\section{REFERENCES}

[1] Benhong, Peng, 2017.Study on Entropy Weight Extension Evaluation of Chain Chain Risk in Cold Chains [J]. Journal of Beijing Jiaotong University (Social Science Edition), 2017,16 (01): 110-119. (2017-01-05) [2017-09-27].

[2] Bingyang, Luo,2015. Weibei study case-based reasoning technology locomotive fault diagnosis system [J]. Wuhan University of Technology (Information \& Management 
Engineering), 2015,37 (01): 38-42. [2017-09-27].

[3] Guangbin, Zhang 2015.Case-based reasoning transmission line single-ended current traveling wave fault location [J]. Proceeding of the CSEE, 2015,35 (06): 1379-1389. [2017-09 27]. DOI: 10.13334 / j.0258-8013.pcsee.2015.06.012.

[4] Jun, Yan, 2017. Application of case reasoning in vehicle fault diagnosis [J]. Application Research of Computers, 2009,26 (10): 3846-3848. [2017-09-27].

[5] Jing, Zheng, 2014. The Similarity Calculation Method of Emergency Cases in Complex Data[J]. Journal of the Chinese Society for Safety Science, 2014,07: 153-158.

[6] Linfa, Li,2015. which text is, Deng Shicong, Lu Kai deep study [J] transformers, fault diagnosis method based on fuzzy 2015,12 three transformers and case matching ratio: 61-66.

[7] Shijiang, Li, 2016. Patient similarity algorithm based on multi-interval interval monitoring and measurement learning
[J]. Journal of Computer Applications, 2016,11: 164-171.

[8] Yingju, Zhang, 2016. Application of Case-based Reasoning Technology in Environmental Emergency Response Decisionmaking [J]. Safety and Environmental Engineering, 2016,01: 94-99.

[9] Shengting, Yang, 2015. who MW, soup sea troubleshooting Mold on Neural Network and case-based reasoning [J] managers, 2015,03: 388-389.

[10] Xianchao, Guo, 2016.Study on product quality and safety risk assessment based on case-based reasoning [J]. Computer \& Automation, 2016,03: 72-75.

[11] Zhanhai, Lu 2012. Comprehensive evaluation of sustainable development of enterprises based on entropy weight method [J]. China Coal, 2012,38 (02): 17-21 + 41. [2017-0927]. 